\title{
Single Atomically Sharp Lateral Monolayer p-n Heterojunction Solar Cells with Extraordinarily High Power Conversion Efficiency
}

$\underline{\text { Meng-Lin Tsai }}$

Ming-Yang Li

José Ramón Durán Retamal

$\underline{\text { Kai-Tak Lam }}$

Yung-Chang Lin

Kazu Suenaga

Lih-Juann Chen

Gengchiau Liang

Lain-Jong Li

$\underline{\mathrm{Jr}-\mathrm{Hau} \mathrm{He}}$

First published: 26 June 2017

https://doi.org/10.1002/adma.201701168

Cited by: 23

UC-eLinks

$\underline{\text { SECTIONS }}$

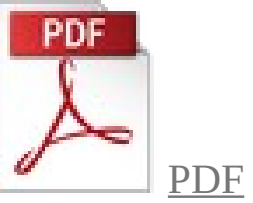

TOOLS

SHARE

Abstract

The recent development of 2D monolayer lateral semiconductor has created new paradigm to develop p-n heterojunctions. Albeit, the growth methods of these heterostructures typically result in alloy structures at the interface, limiting the development for high-efficiency photovoltaic (PV) devices. Here, the PV properties of sequentially grown alloy-free 2D monolayer $\mathrm{WSe}_{2}-$ $\mathrm{MoS}_{2}$ lateral p-n heterojunction are explores. The PV devices show an extraordinary power 
conversion efficiency of $2.56 \%$ under AM 1.5G illumination. The large surface active area enables the full exposure of the depletion region, leading to excellent omnidirectional light harvesting characteristic with only $5 \%$ reduction of efficiency at incident angles up to $75^{\circ}$. Modeling studies demonstrate the PV devices comply with typical principles, increasing the feasibility for further development. Furthermore, the appropriate electrode-spacing design can lead to environment-independent PV properties. These robust PV properties deriving from the atomically sharp lateral p-n interface can help develop the next-generation photovoltaics.

Vertically stacked van der Waals (vdW) 2D monolayer-based materials developed recently have attracted much attention because of their extraordinary electrical and optical properties wellsuited to develop ultrathin electronic devices such as diodes, photodetectors, and solar cells.1$\underline{9}$ Thanks to improvements made in 2D monolayer-based material growth and transfer techniques, all-2D monolayer-based devices that have graphene electrodes, boron nitride dielectric, and transition metal dichalcogenides (TMDs) semiconductors have been successfully vertically stacked, unveiling a new era in nanotechnology research. $\underline{4}$ In fact, vertically stacked vdW 2D monolayer-based heterostructures possessing ultrahigh power densities such as $\mathrm{WS}_{2}$ $\mathrm{MoS}_{2}, \mathrm{WSe}_{2}-\mathrm{MoS}_{2}$, and graphene-MoS have been reported as high-performance 2D monolayerbased PV devices. $\underline{3}, \underline{5}, \underline{6}$ However, the quality of vertically stacked vdW 2D monolayer-based devices is mainly hindered by the complexity of the multiple-step transfer technique and by formation of quenching centers, that is, contaminants and defects at the interface, or corrugations at the surface. 10 -12 Although direct epitaxial growth of 2D monolayer lateral TMDs heterostructures has been proposed to overcome these challenges, the as-fabricated heterostructures are limited to certain metals or chalcogens, and the parameters used to grow these structures yield alloy structures at the interface that inhibit the formation of ideal p-n heterojunctions. $\underline{13}-\underline{15}$ Recently, we reported our success in controlled epitaxial growth of monolayer $\mathrm{WSe}_{2}-\mathrm{MoS}_{2}$ lateral heterojunction that has atomically sharp interface; we achieved this through sequential chemical vapor deposition of $\mathrm{WSe}_{2}$ and $\mathrm{MoS}_{2}$ in two separate furnaces.16

Besides challenges of fabricating and growing 2D monolayer-based materials, also challenging is controlling their electronic properties. Research has demonstrated that gate control of $2 \mathrm{D}$ monolayer p-n heterojunction enables tuning electronic properties.17-22 For example, dual gatetuning (back left and right gates) of monolayer $\mathrm{WSe}_{2}$ enables electrical-doping to develop lightemitting diodes, photodetectors, and solar cells, $\underline{19}-\underline{21}$ and dual gate-tuning (top and back gates) of $\mathrm{WSe}_{2}-\mathrm{MoS}_{2}$ tunneling field effect transistors reduces the switching voltage to smaller values for lower power consumption. 22 However, designing dual gate tuning of vdW 2D monolayer heterostructures-based devices is not only expensive, time consuming, and complex, but also limits the device's active surface area. As a solution, we believe that single-back gate-tuning scheme can be used to tune the electrical properties of atomically sharp 2D monolayer lateral 
heterojunction so that the planar heterojunction can be used to fabricate practical devices without sacrificing the precious active surface area.

The large surface active area of 2D monolayer lateral heterojunction acts as double-edged sword because although it allows full exposure of the heterojunction to light illumination, it is highly sensitive to chemisorption from environmental gases, and thus lowering the stability and performance of PV devices. In fact, the photoelectrical properties of 2D monolayer lateral heterojunctions are expected to be highly gas-sensitive to surface area. Specifically, $\mathrm{O}_{2}$ chemisorption onto chalcogen vacancies of monolayer TMDs reduces electron conductance significantly and usually inhibits the operation of 2D monolayer-based devices.23, 24 Therefore, additional protective layers such as polymethyl methacrylate (PMMA) are typically coated on top of 2D monolayer-based materials to preserve stability and to prevent performance degradation caused by environmental gases. However, protective layers substantially thicken the device and reduce the light absorption within the 2D monolayer-based material, thus degrading device performance. Alternatively, rational design of the electrode spacing and/or the heterojunction interface location (near electrode or at the electrode gap center) can serve to diminish or suppress the effect of chemisorption from environmental gases. 25

Here, we report solar cells based on atomically sharp 2D monolayer $\mathrm{WSe}_{2}-\mathrm{MoS}_{2} \mathrm{p}-\mathrm{n}$ lateral heterojunction synthesized by two-step epitaxial growth. We achieve power conversion efficiency (PCE) up to 2.56\% under AM 1.5G illumination. The planar structure of the cells results in unprecedented omnidirectional light harvesting behavior with merely 5\% loss of PCE at high angles of incidence (AOIs) of $75^{\circ}$. Our low-temperature measurements and simulation of the open-circuit voltage $\left(V_{o c}\right)$ and short-circuit current $\left(I_{\mathrm{sc}}\right)$ confirm the photovoltaic effect originates from the lateral p-n junction formed by a single $\mathrm{WSe}_{2}-\mathrm{MoS}_{2}$ monolayer. Furthermore, our rational electrode-spacing design indicates that environment-independent 2D monolayerbased devices are feasible without protective layers. The results demonstrate the potential of monolayer p-n lateral heterojunction for developing next-generation PV devices.

The 2D lateral $\mathrm{WSe}_{2}-\mathrm{MoS}_{2}$ heterojunction shown in Figure $\underline{1}$ a was epitaxially grown on sapphire substrates by two-step chemical vapor deposition of $\mathrm{WSe}_{2}$ at 925 and $\mathrm{MoS}_{2}$ at $755{ }^{\circ} \mathrm{C}$. After the growth processes, atomic force microscopy (AFM) and scanning transmission electron microscopy (STEM) measurements were performed to characterize the as-grown lateral heterostructures. Figure $1 \mathrm{~b}$ shows the annular dark field image obtained with STEM, indicating the lateral junction featuring with the atomically sharp interface without interdiffusion of atoms, which is vital for preserving optoelectronic/electrical properties driven by the intralayer coupling. These results and the lower-temperature growth of $\mathrm{MoS}_{2}$ than of $\mathrm{WSe}_{2}$ suggest that the 
$\mathrm{MoS}_{2}$ growth is promoted by unsaturated $\mathrm{W}$ dangling bonds at peripheral edges of $\mathrm{WSe}_{2}$ triangular domains where Se atoms are replaced by $\mathrm{S}$ atoms without substitutional atomic interdiffusion or alloying at junction interface. $\underline{13}, \underline{16}$ Figure $\underline{10}$ and Figure S1 (Supporting Information) show the AFM image and height profile analysis across a plotted line of the $\mathrm{WSe}_{2}-$ $\mathrm{MoS}_{2}$ heterostructure, respectively. It is shown that there is almost no height difference between $\mathrm{WSe}_{2}$ and $\mathrm{MoS}_{2}$ regions, suggesting atomically lateral growth of the monolayer heterostructures. This atomically thin and atomically sharp interface of lateral heterojunctions preserves the integrity of the unique optoelectronic properties derived from the intralayer-coupling and represent a clear advantage compared to vertical junctions. For example, conventional CMOS semiconductor junctions developed by ion implantation or thermal diffusion result in unfavorable dopant concentration gradient near the interface that limits the formation of ideal p-n junctions.26 Although vdW 2D monolayer heterostructures developed by vertical stacking are absent of atomic interdiffusion for interlayer coupling, they exhibit significant radiative recombination of spatially indirect excitons that limit PV carrier generation. $\underline{27}$

(a)

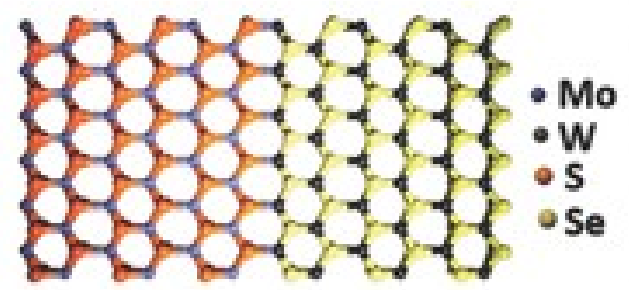

(d) (b)

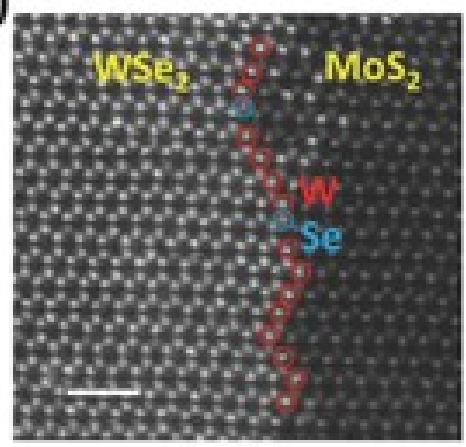

(c)

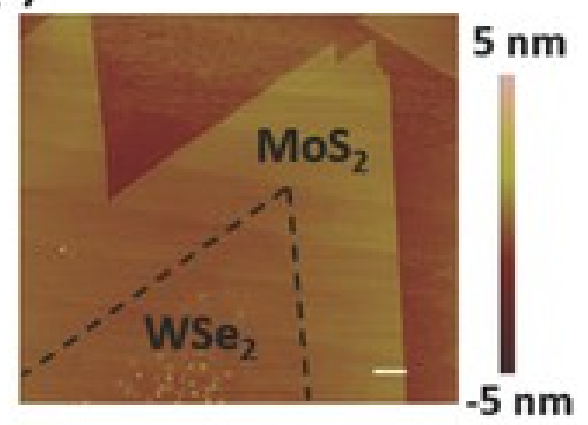

(e)
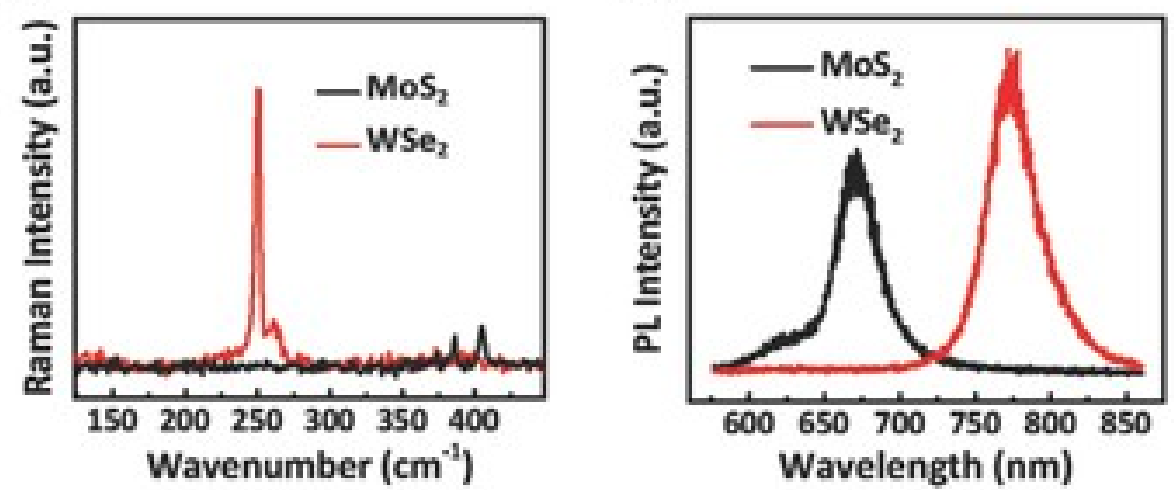

Figure 1

Open in figure viewerPowerPoint

$\mathrm{a}-\mathrm{c}$ ) Schematic, high-resolution STEM image, and AFM image of monolayer $\mathrm{WSe}_{2}-\mathrm{MoS}_{2}$ lateral heterostructure. Scale bar: $1 \mathrm{~nm}$ in (b) and $1 \mu \mathrm{m}$ in (c). d,e) Raman and PL spectra of $\mathrm{MoS}_{2}$ and $\mathrm{WSe}_{2}$ regions. 
To confirm the chemical composition and optical bandgap of 2D monolayer $\mathrm{WSe}_{2}-\mathrm{MoS}_{2}$ lateral heterostructure, we have performed photoluminescence (PL) mapping, Raman mapping, electron energy loss spectroscopy, polarization-resolved second-harmonic generation microscopy, and scanning Kelvin probe microscopy in our previous work.16Here, Raman and PL spectroscopy measurements have been carried out and shown in Figure 1d,e, respectively. The Raman peak at $250 \mathrm{~cm}^{-1}$ measured from the inner triangular domain corresponds to the $\mathrm{A}_{\mathrm{gg}}$ resonant mode of $\mathrm{WSe}_{2}$, and the Raman peaks at 405 and $385 \mathrm{~cm}^{-1}$ measured from the outer triangular domain correspond to the out-of-plane $\mathrm{A}_{1 \mathrm{~g}}$ and in-plane $\mathrm{E}_{2 \mathrm{~g}}$ resonant modes of $\mathrm{MoS}_{2}$, respectively.28, $\underline{29}$ Similarly, the PL peaks at 672 and $778 \mathrm{~nm}$ measured from outer and inner triangular domains correspond to the near-band edge optical emissions of direct bandgap monolayer $\mathrm{MoS}_{2}(\approx 1.8 \mathrm{eV})$ and $\mathrm{WSe}_{2}(\approx 1.6 \mathrm{eV})$, respectively.19, $\underline{30}$ These Raman and PL results are chemical and optical fingerprints that confirm the coexistence of spatially modulated $\mathrm{MoS}_{2}$ and $\mathrm{WSe}_{2}$ triangular domains, corroborating the formation of the 2D monolayer $\mathrm{WSe}_{2}-$ $\mathrm{MoS}_{2}$ lateral heterostructure.

The as-grown 2D monolayer $\mathrm{WSe}_{2}-\mathrm{MoS}_{2} \mathrm{p}-\mathrm{n}$ lateral heterostructures were then transferred onto the $\mathrm{SiO}_{2}(260 \mathrm{~nm}) / \mathrm{Si}$ substrate to fabricate the PV devices. Figure 2a shows schematic, scanning electron microscopy (SEM) image, and energy band diagram of the monolayer devices. Ti/Au $(10 / 50 \mathrm{~nm})$ and $\mathrm{Au}(50 \mathrm{~nm})$ electrodes were patterned by electron beam lithography and deposited by electron beam evaporation to form ohmic contacts with $\mathrm{MoS}_{2}$ and WSe , respectively. Figure S2 (Supporting Information) shows the optical microscopy images of the lateral heterojunction connected in parallel and enlarged high-resolution images of each cell. The active area of the PV device is defined as the summation of the multiplication of electrode width and electrode spacing of the two devices, which are both $2 \mu \mathrm{m} \times 2 \mu \mathrm{m}$. The current-voltage (IV) characteristics under AM 1.5G illumination of the cell 1, cell 2, and cell 1 and cell 2 connected in parallel (cell 1//cell 2) are shown in Figure 2 b. As listed in Table 1 , the two cells connected in parallel yields a $V_{\text {oc }}$ of $0.39 \mathrm{~V}$ and $I_{\mathrm{sc}}$ of $1.27 \mathrm{nA}$, which is almost equal to the sum of each cells measured individually. The PCE of the device was calculated by

$$
\mathrm{PCE}=\frac{V_{\mathrm{oc}} \times I_{\mathrm{sc}} \times \mathrm{FF}}{P \times A} \times 100 \%
$$

where $P$ and $A$ are the power density of the light source and the active area of the device, respectively. Although vertically stacked vdW 2D monolayer-based heterostructures such as graphene- $\mathrm{MoS}_{2}, \mathrm{WSe}_{2}-\mathrm{MoS}_{2}$, and $\mathrm{WS}_{2}-\mathrm{MoS}_{2}$ have been reported as PV devices (shown in Table $\mathrm{S} 1$, Supporting Information), the reported efficiencies of $0.1 \%-1 \%, \approx 0.2 \%$, and $0.4 \%-1.5 \%$, respectively, are still low due to serious radiative recombination of spatially indirect excitons at the vertically stacked vdW interfaces. 27 Alternatively, we believe that our atomically sharp 
interface of the lateral heterostructure minimizes the radiative recombination of vertically stacked heterojunctions, and thus the PCE enhances. To further characterize the PV properties of the device, filling factor (FF) and PCE as a function of illumination intensity of the parallel solar array are presented in Figure 2 c,d, respectively. As the light intensity increases, additional charge carriers are induced, resulting in the enhancement of $I_{\mathrm{sc}}$ and thus PCE. However, increasing the light intensity also comes along with more charge recombination events, resulting in reduced FF and thus PCE. It is noted that in Si and organic-based solar cells that under high-intensity light illumination, excessive charge carriers may accumulate at the electrode-semiconductor interface, inducing charge recombination, increasing series resistance, and lowering the fill factor of the solar cells. $\underline{31}, \underline{32}$ Therefore, back surface field and carrier selective layers have been applied to improve the carrier transport at the electrode-semiconductor interface.33, 34 For 2D materials, the carrier transport at the 2D material-electrode has also been studied to affect the performance and properties of 2D devices significantly. $\underline{35}$, 36 Accordingly, it is shown that under 1 sun (1000 $\mathrm{W} \mathrm{m}^{-2}$ ) condition, the device exhibits the highest PCE of 2.56\%, and this PCE is the highest efficiency illuminated by broadband/solar light source being reported among 2D monolayer lateral heterojunction. We attribute such high PCE to the high quality atomically sharp interface. The parallel circuit design indicates that the device can be effectively devised into large-scale solar modules to achieve performance comparable to conventional solar devices. Additionally, excellent omnidirectional light harvesting behavior has been characterized and shown in Figure 2e. The normalized PCE is defined as the measured PCE divided by the maximum PCE. Our lateral heterojunction-based parallel solar cell shows only a 5\% PCE reduction from $0^{\circ}$ to $75^{\circ} \mathrm{AOI}$, suggesting nearly identical power density at the junction for a wide range of AOIs. We attribute this extraordinary omnidirectional light harvesting ability to the atomically thin and large active area of our 2D monolayer lateral heterojunction-based solar cells where light is highly absorbed from any directions. This omnidirectional property highlights a compelling advantage to conventional vertical solar cells where light is highly scattered or reflected at the top planar surface. For example, Si-based solar cells show $90 \%$ PCE reduction from $0^{\circ}$ to $75^{\circ}$ AOI. 37 To evaluate the stability and reproducibility of the $\mathrm{WSe}_{2}-\mathrm{MoS}_{2}$ lateral heterojunction solar cell, normalized PCE over a $30 \mathrm{~d}$ period and photovoltaic parameters of five different cells have been shown in Figure S3 and Table S2 (Supporting Information), respectively. It is shown that after $30 \mathrm{~d}$, the solar cell retains $70 \%$ of its initial PCE. The result demonstrates the high stability of the $\mathrm{WSe}_{2}-\mathrm{MoS}_{2}$ solar cell for practical applications. The measurement of five different devices with statistical errors has also demonstrated excellent reproducibility of our device fabrication. The $J_{\mathrm{sc}}$ values shown in Table S2 (Supporting Information) are calculated by dividing the $I_{\mathrm{sc}}$ by the active area $(2 \mu \mathrm{m} \times 2 \mu \mathrm{m})$ of the devices. 
(a)

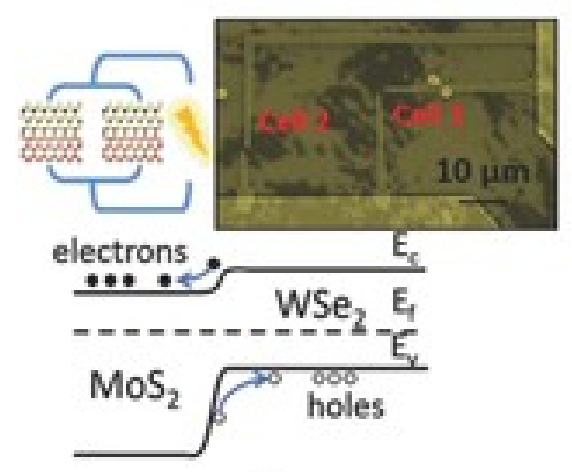

(d) (b)

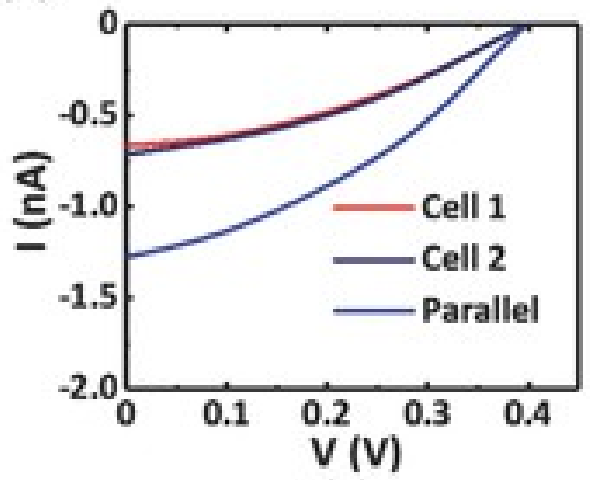

(e) (c)

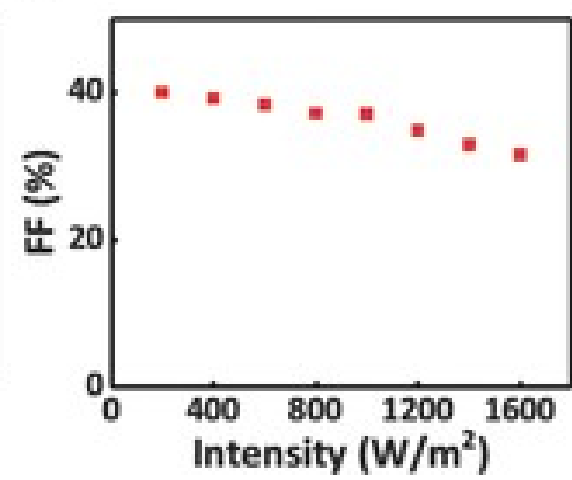

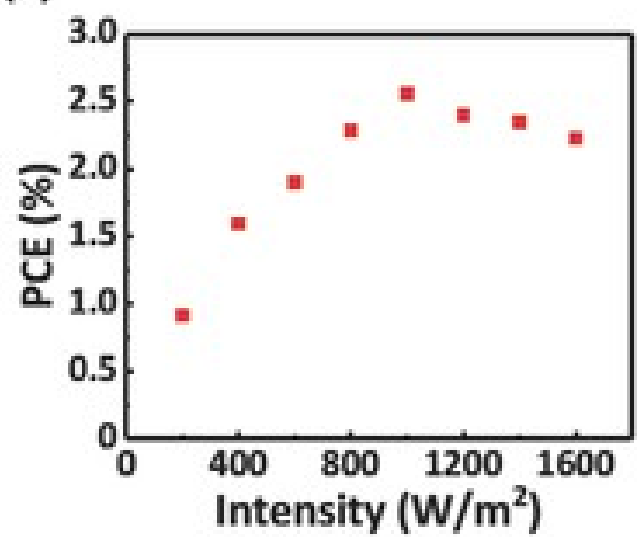

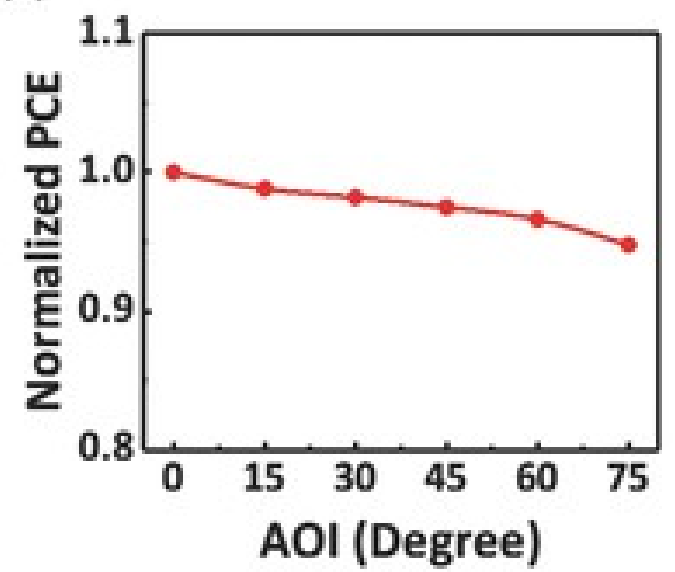

Figure 2

Open in figure viewerPowerPoint

a) Schematic, SEM image, and energy band diagram of 2D monolayer $\mathrm{WSe}_{2}-\mathrm{MoS}_{2} \mathrm{p}-\mathrm{n}$ lateral heterojunction PV devices. b) $I-V$ characteristics of the devices shown in (a) under AM 1.5G light illumination. c,d) Light intensity dependent FF and PCE of the device under AM 1.5G light illumination. e) Angular-dependent normalized PCE of the device under AM 1.5G light illumination.

Table 1. PV properties of the single atomically sharp monolayer $\mathrm{WSe}_{2}-\mathrm{MoS}_{2}$ lateral heterojunction solar cells shown in Figure 2a

Devices

Cell 1

Cell 2
$V_{\text {oc }}[\mathrm{V}]$

0.39
$I_{s c}[n A]$

0.66
FF [\%]

37.55

2.43
PCE [\%]

(20)

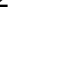
0.39
0.71

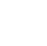




\begin{tabular}{|l|c|c|c|c|}
\hline Devices & $\boldsymbol{V}_{\text {oc }}[\mathbf{V}]$ & $\boldsymbol{I}_{\mathbf{s c}}[\mathbf{n A}]$ & FF [\%] & PCE [\%] \\
Cell 1//cell 2 & 0.39 & 1.27 & 35.84 & 2.21 \\
\hline
\end{tabular}

Next, we validate the PV effect arising from the lateral heterojunction by temperaturedependent $I-V$ measurements. For a typical p-n junction based solar cell, the temperature dependence of $V_{\text {oc }}$ can be calculated according to the reverse saturation current $\left(I_{0}\right)$

$$
I_{0}=q\left(\frac{D_{\mathrm{n}}}{L_{\mathrm{n}} N_{\mathrm{A}}}+\frac{D_{\mathrm{p}}}{L_{\mathrm{p}} N_{\mathrm{D}}}\right) n_{\mathrm{i}}^{2}
$$

where $n_{\mathrm{i}}$ is the intrinsic carrier density $\left(\approx 10^{10} \mathrm{~cm}^{-2}\right.$ for both $\mathrm{WSe}_{2}$ and $\left.\mathrm{MoS}_{2}\right), N_{\mathrm{A}}$ and $N_{\mathrm{D}}$ are densities of acceptor and donor atoms, $D_{\mathrm{n}}$ and $D_{\mathrm{p}}$ are diffusion constants of minority carriers in $\mathrm{n}$ and p regions, and $L_{\mathrm{n}}$ and $L_{\mathrm{p}}$ are diffusion lengths of minority carriers in $\mathrm{n}$ and p regions, respectively. $\underline{38}$ Since $n_{\mathrm{i}}$ can be expressed as

$n_{\mathrm{i}}^{2}=A T^{3} \exp \left(\frac{E_{\mathrm{g}}}{k T}\right)$

where $A$ is a temperature-independent constant, $E_{\mathrm{g}}$ is the material band gap, and $k$ is the Boltzmann constant. $I_{0}$ and $V_{\text {oc }}$ can be expressed as

$$
\begin{aligned}
& I_{0}=B T^{3} \exp \left(-\frac{E_{\mathrm{g}}}{k T}\right) \\
& V_{\propto}=\frac{k T}{q} \ln \left(\frac{I_{\mathrm{sc}}}{I_{0}}\right)_{(5)}
\end{aligned}
$$

By assuming that $\mathrm{d} V_{\text {oc }} / \mathrm{d} T$ does not depend on $\mathrm{d} I_{\mathrm{sc}} / \mathrm{d} T, \mathrm{~d} V_{\mathrm{oc}} / \mathrm{d} T$ can be formulated as

$$
\frac{\mathrm{d} V_{\propto}}{\mathrm{d} T}=\frac{V_{\propto}-V_{\mathrm{g}}}{T}-\frac{3 k}{q}=-\frac{V_{\mathrm{g}}-V_{\propto}+\frac{3 k T}{q}}{T}
$$

where $V_{\mathrm{g}}=E_{\mathrm{g}} / q$. For typical semiconductor such as $\mathrm{Si}, \mathrm{d} V_{\mathrm{oc}} / \mathrm{d} T$ is usually negative based on the above equations and $\mathrm{d} I_{\mathrm{s}} / \mathrm{d} T$ is positive due to decreased bandgap energy $\left(E_{\mathrm{g}}\right)$. Figure $\mathrm{S} 4 \mathrm{a}, \mathrm{b}$ (Supporting Information) shows the temperature-dependent $I-V$ characteristics under dark and AM 1.5G illumination. At high temperatures, the device exhibits higher $I_{\mathrm{sc}}$ and lower $V_{\text {oc }}$ than at low temperatures, which is consistent with those of typical semiconductor p-n junctions. Compared with Schottky junctions, p-n junctions are expected to have strong temperature dependence due to the exponential dependence of the current density on both temperature and 
bandgap energy.39 Therefore, we conclude that the PV effect of the device indeed originates from the $\mathrm{WSe}_{2}-\mathrm{MoS}_{2} \mathrm{p}$-n lateral heterojunction.

Due to the atomically sharp interface of the 2D monolayer-based lateral heterojunction, it is possible to design devices beyond the scaling limit. However, the electrical transport properties are expected to be highly dependent on electrode-spacing because the active area and carrier collection depend on distance. Therefore, we fabricated and simulated field effect transistors with 2, 5, and $7 \mu \mathrm{m}$ electrode spacing to study the electronic transport properties of the lateral heterojunction. In our previous work, we have estimated that the depletion length of the heterojunction is $\approx 320 \mathrm{~nm}$. 16 The exciton diffusion lengths of $\mathrm{MoS}_{2}$ and $\mathrm{WSe}_{2}$ are $\approx 400$ and 160 $\mathrm{nm}$, respectively. 16 Therefore, for the devices fabricated in this work which exhibit heterojunction lengths larger than $2 \mu \mathrm{m}$, the intrinsic device performance without gate bias should be independent of the length of the heterojunction. Figure 3 a and Figure S5 (Supporting Information) show the schematic of electrode spacing design realized in the experiment and the simulation details, respectively. The experimental and simulated $I_{\mathrm{d} s}-V_{\mathrm{g}}$ characteristics under dark at $V_{\mathrm{ds}}=2 \mathrm{~V}$ of these devices revealed different $I_{\mathrm{ds}}$ and dissimilar gate effect, as shown in Figure $\underline{3} \mathrm{~b}$. The $2 \mu \mathrm{m}(7 \mu \mathrm{m})$ electrode spacing device exhibits the highest (lowest) current and the weakest (strongest) gate effect. We attribute these differences to disparity on carrier mobilities and doping levels between the $\mathrm{WSe}_{2}$ and $\mathrm{MoS}_{2}$. It has been reported that $\mathrm{MoS}_{2}$ is an intrinsically moderately n-doped 2D material, whereas $\mathrm{WSe}_{2}$ is an intrinsically weakly p-doped 2D material and exhibits ambipolar behavior. $\underline{6}$ The negligible gate effect and high $I_{\mathrm{ds}}$ of the $2 \mu \mathrm{m}$ electrode spacing device can be attributed to the high injection current caused by the enhanced electric field at such short drain-source distance, hence gate bias ranging from -50 to $50 \mathrm{~V}$ are not sufficient to modulate $I_{\mathrm{ds}}$. Whereas, the relatively low $I_{\mathrm{ds}}$ at $0 \mathrm{~V}$ gate bias of the $7 \mu \mathrm{m}$ electrode-spacing device compared to the 2 or $5 \mu \mathrm{m}$ electrode-spacing devices is caused by the poor electrical conductivity (due to the intrinsically weak doping) of WSe $\mathrm{W}_{2}$. Conversely, at high negative gate bias, the doping concentration and mobility of $\mathrm{WSe}_{2}$ increase, leading to improved p-n junction and higher $I_{\mathrm{ds}}$, while at high positive gate bias, the $\mathrm{WSe}_{2}$ becomes n-type and the mobility increases, leading to n-n junction and higher $I_{\mathrm{d}}$. 


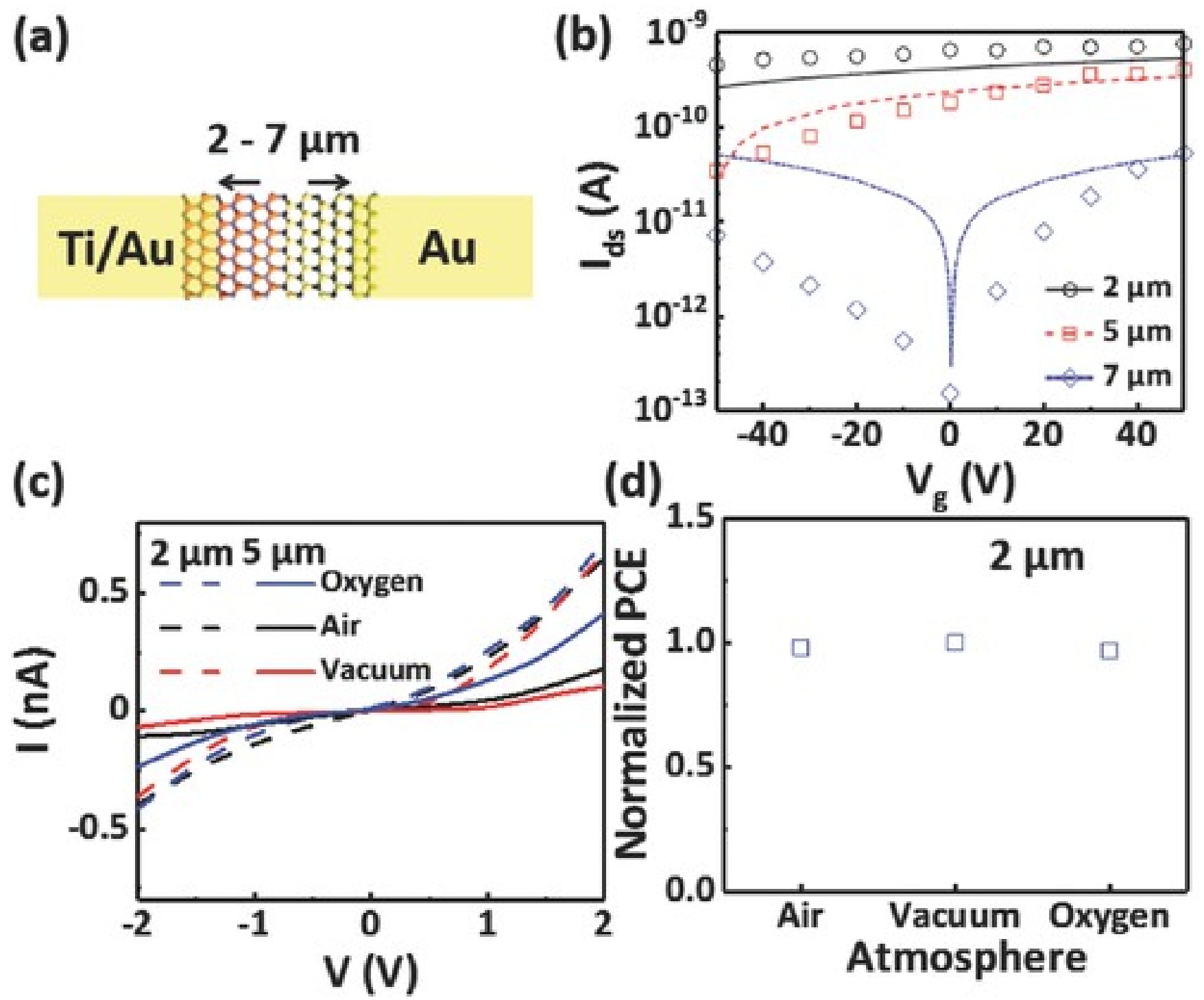

Figure 3

Open in figure viewerPowerPoint

a,b) Schematic, simulated (lines), and experimental (symbols) dark $I_{\mathrm{ds}}-V_{\mathrm{g}}$ characteristics of the monolayer $\mathrm{WSe}_{2}-\mathrm{MoS}_{2}$ lateral heterojunctions at $V_{\mathrm{ds}}=2 \mathrm{~V}$ with different electrode-spacing distances. c) Dark $I-V$ characteristics under various ambient condition for monolayer $\mathrm{WSe}_{2}-$ $\mathrm{MoS}_{2}$ lateral heterojunctions with $2 \mu \mathrm{m}$ (dashed) and $5 \mu \mathrm{m}$ (solid) electrode-spacing distances. d) Normalized PCE of the device under various ambient environments.

Furthermore, the gas doping effect and its dependence on electrode spacing are also of interest in lateral heterojunctions because they can modulate device performance. For example, $\mathrm{O}_{2}$ adsorption on $\mathrm{MoS}_{2}$ defect sites reduces electrical conductivity by trapping electrons. 23 Therefore, we aim to study the influence of the environmental conditions on the electrical transport of the 2D monolayer $\mathrm{WSe}_{2}-\mathrm{MoS}_{2} \mathrm{p}-\mathrm{n}$ lateral heterojunction by measuring the $I-V$ characteristics of the 2 and $5 \mu \mathrm{m}$ electrode-spacing devices under vacuum, air, and $\mathrm{O}_{2}$ rich environments. As shown in Figure $\underline{3}$ c, under air or $\mathrm{O}_{2}$-rich environments, both devices 
exhibit increased current compared to vacuum, although the $2 \mu \mathrm{m}$ electrode-spacing device exhibits lower enhancement and negligible difference between air and $\mathrm{O}_{2}$-rich environments. Although we attribute the increased current to the combined effect of $\mathrm{O}_{2}$ adsorption in $\mathrm{MoS}_{2}$ and WSe $e_{2}$, simulation studies suggest that $\mathrm{O}_{2}$ adsorption interacts more strongly in $\mathrm{WSe}_{2}(1.136$ electrons averaged-trap) than in $\mathrm{MoS}_{2}$ (0.988 electrons averaged-trap). $\underline{2}$ Therefore, $\mathrm{WSe}_{2}$ is expected to become more heavily doped (more p-type), and thus we regard $\mathrm{WSe}_{2}$ as the primary cause of the increased current. However, when we shortened the electrode spacing to $2 \mu \mathrm{m}$, the number of available defect sites decreases, reducing the probability of trapping gas molecules from the environment. Consequently, the current enhancement in air and $\mathrm{O}_{2}$-rich environment becomes less prominent and the device is less affected by environmental gases. To corroborate the environment-independent properties, we further study the PV properties of the $2 \mu \mathrm{m}$ electrode-spacing device under same environmental conditions by measuring the $I-$ $V$ characteristics and calculating the PCEs (shown in Figure $\underline{3} \mathrm{~d}$ ). We obtain relatively constant PCEs under air, vacuum, and $\mathrm{O}_{2}$-rich environments, suggesting that the PV properties of the narrow $(2 \mu \mathrm{m})$ electrode-spacing device are also independent of environmental gases. This serves not only to maintain high PV performance under different environmental conditions but also to avoid further passivation layers that complicate the fabrication process and limit the light absorption of the underlying monolayers. We also foresee that further electrode-spacing reduction down to nanometer scale can eventually lay foundation for defect-free lateral heterojunctions that are fully independent of environmental gases.

In summary, we demonstrate a successful fabrication of atomically sharp 2D monolayer $\mathrm{WSe}_{2}$ $\mathrm{MoS}_{2}$ p-n lateral heterojunction-based solar cells. We achieved the PCE of 2.56\% under AM 1.5G by exploiting the uniqueness of the atomically sharp interface of the planar heterojunction. Remarkably, we achieved unprecedented omnidirectional light harvesting capability with $95 \%$ of efficiency maintained at high AOIs up to 75\%; this efficiency is unattainable for traditional vertical junction solar cells. The $V_{\mathrm{oc}}$ and $I_{\mathrm{sc}}$ temperature dependence done by experiments and simulation is well explained by using typical p-n junction principles. Finally, by optimizing the electrode spacing, we achieved gate-tuning controllability and environment-independent PCE. These intriguing characteristics we report here will lead to even more widespread use of monolayer lateral heterojunctions for next-generation electronics and photovoltaics.

\section{Experimental Section}

Growth and Characterization of Monolayer $\mathrm{WS}_{2}-\mathrm{MoS}_{2}$ Lateral Heterostructures: Single-crystal WSe $e_{2}$ monolayer was first grown by placing $\mathrm{WO}_{3}$ powders in a quartz boat at the heating zone 
center of the furnace. The sapphire substrate was placed at the downstream side next to the quartz boat. Se powders were placed in a separate quartz boat at the upper stream side of the furnace and the temperature was maintined at $260{ }^{\circ} \mathrm{C}$ during the reaction. Ar/ $/ \mathrm{H}_{2}$ flow was controlled at $\mathrm{Ar}=90 \mathrm{sccm}$ and $\mathrm{H}_{2}=6 \mathrm{sccm}$ with the chamber pressure of 20 Torr. The heating zone was heated to $925^{\circ} \mathrm{C}$, kept for $15 \mathrm{~min}$, and cooled down to room temperature. Then the sample was moved to a separate furnace. The $\mathrm{MoS}_{2}$ synthesis was carried out by switching the sources to $\mathrm{MoO}_{3}$ and S powders. The Ar flow was controlled at 70 sccm with the chamber pressure of 40 Torr. The center zone and S source were heated to 725 and $190{ }^{\circ} \mathrm{C}$, respectively, and kept for $15 \mathrm{~min}$. Finally, the sample was cooled down to room temperature. Surface morphology and surface potential of the heterostructures were characterized using a commercial atomic force microscope (Cypher ES - Asylum Research Oxford Instruments). Raman spectra were collected in a Witec alpha 300 confocal Raman microscopic system including RayShield coupler with exciting laser wavelength of $532 \mathrm{~nm}$. The laser spot size is around $0.5 \mu \mathrm{m}$. Raman signal was collected by a $100 \times$ objective lens (NA = 0.9) from Carl Zeiss Microscopy GmbH and dispersed by a 1800 lines $\mathrm{mm}^{-1}$ grating for Raman measurement and a 300 lines $\mathrm{mm}^{-1}$ grating for PL measurements. PL measurement was also performed in the Witec alpha 300 confocal system.

Fabrication and Characterization of Devices: The monolayer $\mathrm{WSe}_{2}-\mathrm{MoS}_{2}$ lateral heterojunction was first transferred onto a $\mathrm{SiO}_{2}(260 \mathrm{~nm}) / \mathrm{Si}$ substrate by a PMMA (950K A4, MicroChem) assisted method. The monolayer $\mathrm{WSe}_{2}-\mathrm{MoS}_{2}$ lateral heterojunction-based device was then defined with electron-beam lithography in a SEM system (JEOL JSM-7001F). Ti/Au (10/50 nm) and $\mathrm{Au}(50 \mathrm{~nm})$ were deposited using electron beam evaporation. $50 \mathrm{~nm}$ of $\mathrm{SiO}_{2}$ was deposited on $\mathrm{MoS}_{2}$ to prevent direct contact of Au with the outer $\mathrm{MoS}_{2}$. After the deposition of the electrodes, the sample was annealed at $300{ }^{\circ} \mathrm{C}$ for $30 \mathrm{~s}$ in a ULVAC MILA-5000 rapid thermal annealer. The measurements were carried out by placing the sample in a Lakeshore probe-station (PS-100) using a certified Class B AM 1.5G solar light source (which exhibits homogeneous and steady light with a power density of $1000 \mathrm{~W} \mathrm{~m}^{-2}$ ) and a Keithley 4200 source meter.

Device Simulation Model: The current characteristics of the 2D monolayer $\mathrm{WSe}_{2}-\mathrm{MoS}_{2}$ lateral heterojunction were simulated with the drift-diffusion model coupled with a 2D Poisson solver, implemented in the finite element solver COMSOL 3.5a. A schematic of the device simulated is shown in Figure S2 (Supporting Information) and the governing Poisson and current continuity equations are, respectively

$$
\begin{aligned}
& \nabla \cdot\left(\varepsilon_{0} \varepsilon_{\mathrm{r}} \nabla \varphi\right)=q\left(n-p+N_{\mathrm{A}}-N_{\mathrm{D}}\right) \\
& \nabla \cdot J_{\mathrm{n}}=\nabla \cdot\left(-q n \mu_{\mathrm{n}} \nabla \varphi+q D_{\mathrm{n}} \nabla n\right)=q R_{\mathrm{n}}
\end{aligned}
$$


$\nabla \cdot J_{\mathrm{p}}=\nabla \cdot\left(-q n \mu_{\mathrm{p}} \nabla \varphi-q D_{\mathrm{p}} \nabla p\right)=-q R_{\mathrm{p}}$

The relative permittivity $\varepsilon_{\mathrm{r}}$ is 4 for both materials and the electron and hole concentrations nand $p$ are calculated based on the electron dispersions of the monolayer 2D materials with respect to the potential profile $\phi . N_{\mathrm{A}}$ and $N_{\mathrm{D}}$ are the acceptor and donor concentrations and $q$ is the elementary charge constant. $J, \mu, D$, and $R$ are the current density, mobility, diffusion coefficient, and recombination rates for the different carriers, respectively. More specifically, the mobility of the material is set at $14 \mathrm{~cm}^{2} \mathrm{~V}^{-1} \mathrm{~s}^{-1}, D=k_{\mathrm{B}} T \mu / q$ with $k_{\mathrm{B}}$ being the Boltzmann constant and $T=300 \mathrm{~K}$, and the significant recombination mechanism is ShockleyRead-Hall, that is, $R \approx n p / \tau(n+p)$ with $\tau$ being the carrier lifetime of $50 \mathrm{ps}$. Additionally, the work function of $\mathrm{MoS}_{2}$ is $0.675 \mathrm{eV}$ lower than that of $\mathrm{WSe}_{2}$ which is included in the simulation model.

\section{Acknowledgements}

This research was supported by KAUST baseline funding.

\section{Conflict of Interest}

The authors declare no conflict of interest. 